Rev. Adm. Saúde - Vol. 18, № 71, abr. - jun. 2018

http://dx.doi.org/10.23973/ras.71.104

ARTIGO ORIGINAL

\title{
Percepção dos profissionais de saúde sobre os fatores associados à subnotificação no Sistema Nacional de Agravos de Notificação
}

Perception of health professionals about the factors associated with underreporting in the National System of Notification Diseases

\section{Maria Aparecida de Souza Melo', Marília Ferreira Dela Coleta², José Augusto Dela Coleta ${ }^{3}$, José Clecildo Barreto Bezerra ${ }^{4}$, Ana Maria de Castro $^{5}$, Ana Luísa de Souza Melo ${ }^{6}$, Ricardo Antonio Gonçalves Teixeira ${ }^{7}$, Daniel Batista Gomes ${ }^{8}$, Huilma Alves Cardoso ${ }^{9}$}

1. Administradora, doutora em medicina tropical e saúde pública. Professora do Campus Anápolis de Ciências Socioeconômicas e Humanas da Universidade Estadual de Goiás, Anápolis GO.

2. Psicóloga, doutora em psicologia. Professora aposentada da Universidade Federal de Uberlândia, Uberlândia MG.

3. Psicólogo, doutor em psicologia social. Professor aposentado da Universidade Federal de Uberlândia, Uberlândia MG.

4. Biólogo, doutor em ciências naturais. Professor e diretor do Instituto de Patologia Tropical e Saúde Pública da Universidade Federal de Goiás, Goiânia GO

5. Biomédica, doutora em parasitologia. Professora do Instituto de Patologia Tropical e Saúde Pública da Universidade Federal de Goiás, Goiânia GO.

6. Advogada. Mestranda em direitos humanos na Universidade Federal de Goiás, Goiânia GO.

7. Matemático e pedagogo, doutor em educação. Professor da Faculdade de Educação da Universidade Federal de Goiás, Goiânia GO.

8. Administrador e contador, mestre em saúde coletiva. Coordenador de Sistemas de Informação em Saúde da Superintendência de Vigilância em Saúde do Estado de Goiás, Goiânia GO.

9. Enfermeira, especialista em saúde pública. Coordenadora do Centro de Referência em Saúde do Trabalhador da Superintendência de Vigilância em Saúde do Estado de Goiás, Goiânia GO. 


\section{RESUMO}

Objetivo: Identificar fatores que contribuem para a subnotificação de doenças e agravos de notificação compulsória no Sistema Nacional de Agravos de Notificação (Sinan), na percepção dos profissionais de saúde. Métodos: Estudo conduzido com base na Técnica dos Incidentes Críticos para a coleta dos dados e na análise de conteúdo, em uma amostra estratificada de trabalhadores e gestores das unidades de vigilância em saúde responsáveis pelas notificações de doenças e agravos de notificação compulsória.

Resultados: Os casos relatados associaram-se a 33 doenças e agravos de notificação compulsória e os principais comportamentos resultantes foram a não notificação ou a notificação tardia no Sinan. A conduta do médico ou enfermeiro, as dificuldades no processo de notificação e os problemas relacionados ao paciente e/ou familiares foram os principais fatores associados à subnotificação. Conclusão: A subnotificação é uma realidade vivenciada nas unidades de atendimento ao paciente e esconde a real situação de saúde da população.

Palavras-chave: vigilância epidemiológica, sistemas de informação em saúde, notificação compulsória, notificação de doenças.

\section{ABSTRACT}

Objective: To identify factors that contribute to the underreporting of diseases and compulsory notification harms in the National System of Notification Diseases (Sinan), in the perception of health professionals. Methods: A conducted study based on the Critical Incidents Technique for data collection and content analysis, in a stratified sample of workers and managers of the health surveillance units responsible for notifying diseases and notifiable harms. Results: The reported cases were associated with 33 diseases and compulsory notification harms and the main behaviors were non-notification or late notification in Sinan. The doctor's and nurse's conduct, difficulties in the notification process, and problems related to the patient and / or family were the main factors associated with underreporting. Conclusion: Underreporting is a reality experienced in patient care units and hides the real health situation of the population.

Keywords: epidemiological surveillance, health information systems, mandatory reporting, disease notification. 


\section{INTRODUÇÃO}

O Sistema Nacional de Vigilância Epidemiológica (SNVE) foi criado no Brasil, por recomendação da $5^{\text {a }}$ Conferência Nacional de Saúde, ocorrida em 1975, e teve como marco a institucionalização das ações de vigilância da Varíola que apoiaram e fomentaram a estruturação de unidades de vigilância epidemiológica no âmbito das secretarias estaduais de saúde. Em 1969 foi organizado, pela Fundação de Serviços de Saúde Pública (FSESP), o primeiro sistema de notificação semanal de doenças que gerava um boletim epidemiológico de circulação quinzenal, processo este que desencadeou ações de controle de doenças evitáveis por imunizações, como o controle da Poliomielite ${ }^{1}$.

A partir da institucionalização, pela Constituição Federal do Brasil de 1988, da promoção, proteção e recuperação da saúde como dever do Estado e direito do Cidadão², o Sistema Único de Saúde (SUS), instituído pela Lei oㅡ 8.080, de 1990 de setembro de $1990^{3}$, incorporou o SNVE e esta reorganização ampliou o conceito de vigilância epidemiológica. Esta passou a ser entendida como "um conjunto de ações que proporciona o conhecimento, a detecção ou prevenção de qualquer mudança nos fatores determinantes e condicionantes da saúde individual ou coletiva, com a finalidade de recomendar e adotar as medidas de prevenção e controle das doenças ou agravos" ${ }^{3}$. Os serviços e ações de saúde no Brasil passaram a ser executados pelas instituições que compõem o SUS ou por aquelas pertencentes à iniciativa privada, organizadas regionalmente e hierarquizadas por níveis de complexidade, segundo as competências e atribuições definida para a União, Estados, Distrito Federal e Municípios ${ }^{3}$.

Para atender aos desafios enfrentados pelo país no que diz respeito a uma situação de saúde complexa e de causalidades distintas, as ações de vigilância epidemiológica foram assumidas pela Secretaria de Vigilância em Saúde (SVS), do Ministério da Saúde (MS), através do Decreto no 4.726/20034. O MS estabeleceu ao SNVE, dentre outras atribuições, a de notificação compulsória, pelos profissionais de saúde ou responsáveis pelos estabelecimentos de saúde públicos ou privados, a ocorrência ou suspeita de doenças e agravos ou eventos de saúde pública relacionados em portaria, em lista atualizada oportunamente ${ }^{5}$. As notificações e investigações dos casos identificados são registradas no Sistema Nacional de Agravos de Notificação (Sinan), criado para operacionalizar tal processo em um nível de gestão o mais próximo possível das unidades notificadoras 6 .

As notificações no Sinan devem ser realizadas de maneira correta e com a frequência estabelecida para permitir que as informações sejam utilizadas para a tomada de decisão sobre as ações de vigilância em saúde, o que nem sempre ocorre ${ }^{1}$. As subnotificações comprometem o planejamento das ações de prevenção e controle epidemiológico em função das estimativas das magnitudes das doenças basearem em dados não fidedignos da realidade epidemiológica ${ }^{7,8}$.

Estudos pontuais são identificados para investigar os índices de subnotificação de doenças e agravos no Sinan e apontam percentuais que diferem em termos do agravo ou doença ou da época e local em que foram investigados, dentre os 
quais muitos considerados de controle prioritário. São escassos os estudos identificados na literatura que abordem objetivamente os fatores associados às subnotificações no Sinan, pois somente três publicações nos últimos dez anos descreveram veladamente os fatores associados à subnotificação da doença ou agravo investigados ${ }^{9-11}$. A subnotificação tem sido associada a diversas causas, tais como aos problemas no diagnóstico e na identificação dos $\operatorname{casos}^{9}$; complexidades das doenças ou agravos ${ }^{9}$; rotinas e protocolos dos serviços ${ }^{9}$; capacidade técnica dos recursos humanos ${ }^{10}$; não valorização da Vigilância Epidemiológica ${ }^{11}$, dentre outras. É importante averiguar, no contexto do trabalho dos profissionais de saúde, as dificuldades, vivenciadas no cotidiano do atendimento aos pacientes, que contribuem para a não notificação de doenças e agravos de notificação compulsória no Sinan.

Este estudo objetivou identificar fatores que contribuem para a subnotificação de doenças e agravos de notificação compulsória no Sistema Nacional de Agravos de Notificação (Sinan), na percepção dos profissionais de saúde.

\section{MÉTODOS}

Abordagens de natureza qualitativa e quantitativa ${ }^{12}$ e, quanto aos objetivos, exploratória ${ }^{13}$, descritiva ${ }^{14}$ e explicativa ${ }^{15}$, baseando-se na Técnica dos Incidentes Críticos $^{16}$ para a coleta dos dados e na análise de conteúdo ${ }^{17,18}$.

A amostragem estratificada ${ }^{19}$ compôs-se de trabalhadores e gestores dos serviços das unidades de Vigilância em Saúde (VISA) municipais do Estado de Goiás, responsáveis pelas notificações de doenças e agravos de notificação compulsória ao Sinan. Na definição da amostra, realizou-se um levantamento sobre a forma de estruturação da VISA nos municípios goianos, com a adesão de 107 (43,5\%) municípios. Selecionaram-se 17 municípios, dentre aqueles que aderiram ao estudo, levando em conta a forma de estruturação da VISA, a participação de pelo menos um município por região de saúde, com diferentes classes populacionais ${ }^{20}$. A cobertura estimada abrangida pelos municípios amostrais representou $41 \%$ da população do Estado de Goiás, fator que torna o estudo significativo. A Tabela 1 apresenta os municípios amostrais segundo a região de saúde, o quantitativo das entrevistas realizadas e respectiva população estimada.

Tabela 1. Distribuição dos municípios amostrais segundo as regiões de saúde de Goiás, quantitativo de entrevistas e população estimada $2010(n=17)$.

\begin{tabular}{l|l|r|r}
\hline Município amostral & Região de Saúde & Entrevistas & População \\
& & (n) & (n) \\
\hline Adelândia & Oeste II & 1 & 2.477 \\
\hline Alto Paraíso de Goiás & Entorno Norte & 3 & 6.885 \\
\hline
\end{tabular}




\begin{tabular}{|c|c|c|c|}
\hline Município amostral & Região de Saúde & $\begin{array}{r}\text { Entrevistas } \\
\text { (n) }\end{array}$ & $\begin{array}{r}\text { População } \\
\text { (n) }\end{array}$ \\
\hline Anápolis & Pirineus & 12 & 334.613 \\
\hline Aparecida de Goiânia & Centro Sul & 12 & 455.657 \\
\hline Bom Jesus de Goiás & Sul & 4 & 20.727 \\
\hline Caldas Novas & Estrada de Ferro & 4 & 70.473 \\
\hline Campinaçu & Norte & 4 & 3.656 \\
\hline Campinorte & Serra da Mesa & 3 & 11.111 \\
\hline Cavalcante & Nordeste I & 3 & 9.392 \\
\hline Goiânia & Central & 16 & 1.302 .001 \\
\hline Itaberaí & Rio Vermelho & 4 & 35.371 \\
\hline Mineiros & Sudoeste II & 4 & 52.935 \\
\hline Moiporá & Oeste I & 1 & 1.763 \\
\hline Novo Gama & Entorno Sul & 3 & 95.018 \\
\hline Quirinópolis & Sudoeste I & 5 & 43.220 \\
\hline Rianápolis & São Patrício & 2 & 4.566 \\
\hline São Domingos & Nordeste II & 2 & 11.272 \\
\hline Total & & 83 & 2.461 .137 \\
\hline \multicolumn{3}{|l|}{ População de Goiás } & 6.003 .788 \\
\hline Percentual amostral (\%) & & & 41,0 \\
\hline
\end{tabular}

Fonte: dados da pesquisa e Instituto Brasileiro de Geografia e Estatística ${ }^{20}$.

Notas: 1) A região de saúde São Patrício foi desmembrada em São Patrício I e II posteriormente à realização da coleta de dados de campo. Assim, Rianápolis representou essas duas regiões na configuração atual; 2) O número de entrevistas (exceto nos municípios de Anápolis, Goiânia e Aparecida de Goiânia em que a amostra foi estratificada por regiões/unidades de atendimento) correspondeu ao total de trabalhadores e gestores dos serviços de saúde responsáveis pelas notificações de doenças e agravos de notificação compulsória ao Sinan. 
O instrumento de coleta de dados de campo foi elaborado com a participação de gestores da Superintendência de Vigilância em Saúde da Secretaria de Saúde do Estado de Goiás e submetido à análise de conteúdo e teste para validação semântica. Este se constituiu de um questionário base para construção do roteiro de entrevista estruturada, com o objetivo de levantar os fatores facilitadores e dificultadores na notificação de doenças e agravos de notificação compulsória, inspirado nos postulados da Técnica dos Incidentes Críticos (TIC). A TIC consiste em elaborar um questionário para a entrevista que contenha a melhor pergunta a ser respondida pelos participantes, composto de questões adaptadas aos objetivos e propósitos da pesquisa ${ }^{16}$. A coleta de campo nas unidades de trabalho dos voluntários dos serviços de VISA, realizada de agosto/2014 a março/2015, precedeu da adoção de métodos com vistas a garantir o sigilo das informações, em atendimento ao disposto na Resolução no 466/2012, do Conselho Nacional de Saúde, especialmente quanto ao uso do Termo de Consentimento Livre e Esclarecido ${ }^{21}$.

Uma análise de conteúdo, na perspectiva de Franco $^{17}$ e Bardin $^{18}$, foi realizada nos relatos colhidos nas entrevistas, o que possibilitou a construção de categorias de análise, que se deu a partir da utilização do software para análise qualitativa NVivo, versão 11. Buscou-se identificar, através dos casos relatados, os comportamentos e as consequências advindas de cada situação. As "situações" são as doenças e agravos identificados nos pacientes atendidos nas unidades de saúde e os "comportamentos" são as ações descritas pelos entrevistados em cada situação que levaram à notificação ou à subnotificação no Sinan. Não se tratavam, portanto, de situações pessoais e sim de narrativas dos profissionais responsáveis pelas notificações que participaram das entrevistas. No processo de análise, os dados foram organizados conforme aproximações de ideias-chave, convergindo em subcategorias e, a posteriori, em categorias e dimensões. Concluída a etapa de categorização das entrevistas, em atendimento aos postulados de Dela Coleta e Dela Coleta ${ }^{16}$, esta organização dos dados foi submetida à apreciação de juízes, constituída por dois pesquisadores referenciais na área da saúde pelo uso desta metodologia, para validação e correção, como forma de minimizar os níveis de subjetividade nesta fase do estudo.

Após a etapa de análise qualitativa dos dados, para a apresentação dos resultados em formato de gráficos e tabelas, utilizou-se como suporte o pacote SPSS (Statistical Package for the Social Sciences), versão 23. Neste artigo serão apresentados os resultados gerais do estudo e os principais fatores que contribuem para a subnotificação de doenças e agravos de notificação compulsória no Sinan, identificados nas situações e comportamentos relatados pelos entrevistados como dificultadores no processo de notificação no Sinan e respectivas categorias e subcategorias de análise.

O projeto foi aprovado pelo Comitê de Ética em Pesquisa do Hospital de Urgência de Goiânia, com o Parecer no 653.323, de 30/05/2014, e teve apoio financeiro da Fundação de Amparo à Pesquisa do Estado de Goiás (FAPEG), Chamada Pública no 12/2013 - Programa de Pesquisa para o Sistema Único de Saúde (PPSUS), Processo no 201410267000320. 


\section{RESULTADOS}

Os 83 sujeitos entrevistados, trabalhadores e gestores dos serviços das unidades de Vigilância em Saúde (VISA) dos 17 municípios amostrais eram, em sua maioria, do sexo feminino (92,8\%), com idade entre 26 e 30 anos $(31,3 \%)$, casados $(48,2 \%)$, com pós-graduação completa $(60,2 \%)$, servidores efetivos do município $(53,0 \%)$, entre 1 e 5 anos de atuação na área da VISA $(39,8 \%)$, exerciam atividades de coordenação $(79,5 \%)$ e atuavam em distintas áreas de atendimento ao paciente (Cais $=13,3 \% ; \mathrm{CClH}=3,6 \%$, Centro de saúde $=6,0 \%$; Cerest $=2,4 \%$; Ciams $=1,2 \%$; ESF $=21,7 \%$; Vigilância Epidemiológica $=25,3 \%$; Hospital municipal $=9,6 \%$; Laboratório $=1,2 \%$; Atendimento de emergência $=3,6 \%$; Núcleo de Vigilância $=9,6 \%$; e Programas de controle de doenças $=2,4 \%$ ).

As entrevistas resultaram no relato de 111 casos ou situações facilitadoras e 133 casos ou situações dificultadoras relacionados ao processo de notificação no Sinan, associados a 33 doenças ou agravos de notificação compulsória (Tabela 2). As doenças ou agravos de notificação compulsória com maior quantidade de menções foram, por ordem decrescente, a Dengue $(n=44)$, Acidente por animal potencialmente transmissor da Raiva $(n=23)$, Hanseníase $(n=20)$ e Sífilis $(n=4)$. Em se tratando das situações dificultadoras, a Dengue $(n=27)$ também apareceu em primeiro lugar, seguida da Hanseníase e da Sífilis (ambas com 9 casos) e, depois, as Hepatites e a Síndrome Respiratória Aguda (com 8 casos cada). Sobre os comportamentos resultantes das situações dificultadoras, $56,4 \%$ dos casos relatados não foram notificados, $21,1 \%$ foram notificados tardiamente e $22,5 \%$ notificados, mas não encerrados no Sinan. Já nas situações facilitadoras, $46,9 \%$ dos casos tiveram a notificação realizada pela enfermagem, 25,2\% pelos profissionais de saúde de maneira geral, 19,8\% pela Vigilância Epidemiológica ou pela Comissão de Controle de Infecção Hospitalar, 4,5\% realizada pelo médico e 3,6\% somente foram identificados por meio de busca ativa realizada nas unidades de saúde e em prontuários.

Tabela 2. Casos ou situações facilitadoras $(n=111)$ e dificultadoras $(n=133)-$ doenças e agravos - relatados nas entrevistas $(n=83)$.

\begin{tabular}{l|r|r|r|r}
\hline Situação & $\begin{array}{r}\text { Casos } \\
\text { Facilitadores } \\
\text { (n) }\end{array}$ & $\begin{array}{r}\text { Casos } \\
\text { Dificultadores } \\
\text { (n) }\end{array}$ & $\begin{array}{r}\text { Total } \\
\text { (n) }\end{array}$ & $\begin{array}{r}\text { Total (\%) } \\
\hline \text { Acidente com material biológico }\end{array}$ \\
\hline Acidente de Trabalho & 3 & 6 & 9 & 3,7 \\
\hline Acidente por animal peçonhento & 6 & 1 & 7 & 2,9 \\
\hline $\begin{array}{l}\text { Acidente por animal potencialmente } \\
\text { transmissor da Raiva }\end{array}$ & 16 & 7 & 23 & 9,4 \\
\hline
\end{tabular}




\begin{tabular}{|c|c|c|c|c|}
\hline Situação & $\begin{array}{r}\text { Casos } \\
\text { Facilitadores } \\
\text { (n) }\end{array}$ & $\begin{array}{r}\text { Casos } \\
\text { Dificultadores } \\
\text { (n) }\end{array}$ & $\begin{array}{r}\text { Total } \\
(n)\end{array}$ & Total (\%) \\
\hline Agravos - violências interpessoais & - & 5 & 5 & 2,0 \\
\hline Coqueluche & 3 & 5 & 8 & 3,3 \\
\hline Dengue & 17 & 27 & 44 & 18,0 \\
\hline Doença de Chagas & 1 & - & 1 & 0,4 \\
\hline Doença Diarreica Aguda & 2 & 3 & 5 & 2,0 \\
\hline $\begin{array}{l}\text { Doença Exantemática (Sarampo e } \\
\text { Rubéola) }\end{array}$ & - & 1 & 1 & 0,4 \\
\hline $\begin{array}{l}\text { Doenças Sexualmente Transmissíveis } \\
\text { (Interesse Estadual) }\end{array}$ & 3 & 2 & 5 & 2,0 \\
\hline Ebola & - & 1 & 1 & 0,4 \\
\hline Eventos Adversos Graves & 2 & 1 & 3 & 1,2 \\
\hline Esquistossomose & - & 1 & 1 & 0,4 \\
\hline Hanseníase & 11 & 9 & 20 & 8,2 \\
\hline Hantavirose & - & 1 & 1 & 0,4 \\
\hline Hepatites & 4 & 8 & 12 & 4,9 \\
\hline HIV-aids & 3 & 2 & 5 & 2,0 \\
\hline Intoxicações Exógenas & - & 4 & 4 & 1,6 \\
\hline Leishmaniose & 2 & 3 & 5 & 2,0 \\
\hline Leptospirose & - & 1 & 1 & 0,4 \\
\hline Malária & - & 1 & 1 & 0,4 \\
\hline Meningites & 7 & 3 & 10 & 4,1 \\
\hline Raiva & 2 & 5 & 7 & 2,9 \\
\hline Sífilis & 10 & 9 & 19 & 7,8 \\
\hline Síndrome Respiratória Aguda (SRAG) & 5 & 8 & 13 & 5,3 \\
\hline Tétano Neonatal & - & 1 & 1 & 0,4 \\
\hline
\end{tabular}




\begin{tabular}{|c|c|c|c|c|}
\hline Situação & $\begin{array}{r}\text { Casos } \\
\text { Facilitadores } \\
\text { (n) }\end{array}$ & $\begin{array}{r}\text { Casos } \\
\text { Dificultadores } \\
\text { (n) }\end{array}$ & $\begin{array}{r}\text { Total } \\
\text { (n) }\end{array}$ & Total (\%) \\
\hline Toxoplasmose & 1 & 1 & 2 & 0,8 \\
\hline Tuberculose & 6 & 5 & 11 & 4,5 \\
\hline Varicela & 1 & 5 & 6 & 2,5 \\
\hline Varíola & 1 & - & 1 & 0,4 \\
\hline $\begin{array}{l}\text { Violência doméstica e outras } \\
\text { violências }\end{array}$ & 2 & 5 & 7 & 2,9 \\
\hline $\begin{array}{l}\text { Violência Sexual e tentativas de } \\
\text { suicídio }\end{array}$ & 3 & - & 3 & 1,2 \\
\hline Total & 111 & 133 & 244 & 100,0 \\
\hline
\end{tabular}

As situações dificultadoras nos processos de notificação de doenças e agravos de notificação compulsória no Sinan foram sistematizadas na dimensão "Percepções dos entrevistados sobre os fatores associados às situações dificultadoras" que resultaram em 14 categorias de análise. Nessas categorias foram inseridas 113 subcategorias associadas às situações dificultadoras que somaram 958 menções nas entrevistas (Figura 1). A conduta do médico ou do enfermeiro $(23,3 \%)$ foi o item com maior número de menções nas entrevistas como fator dificultador no processo de notificação, seguida das dificuldades no processo de notificação $(13,2 \%)$, dos problemas relacionados ao paciente e/ou familiares $(12,9 \%)$ e daqueles relacionados ao diagnóstico da doença ou agravo $(8,8 \%)$. 


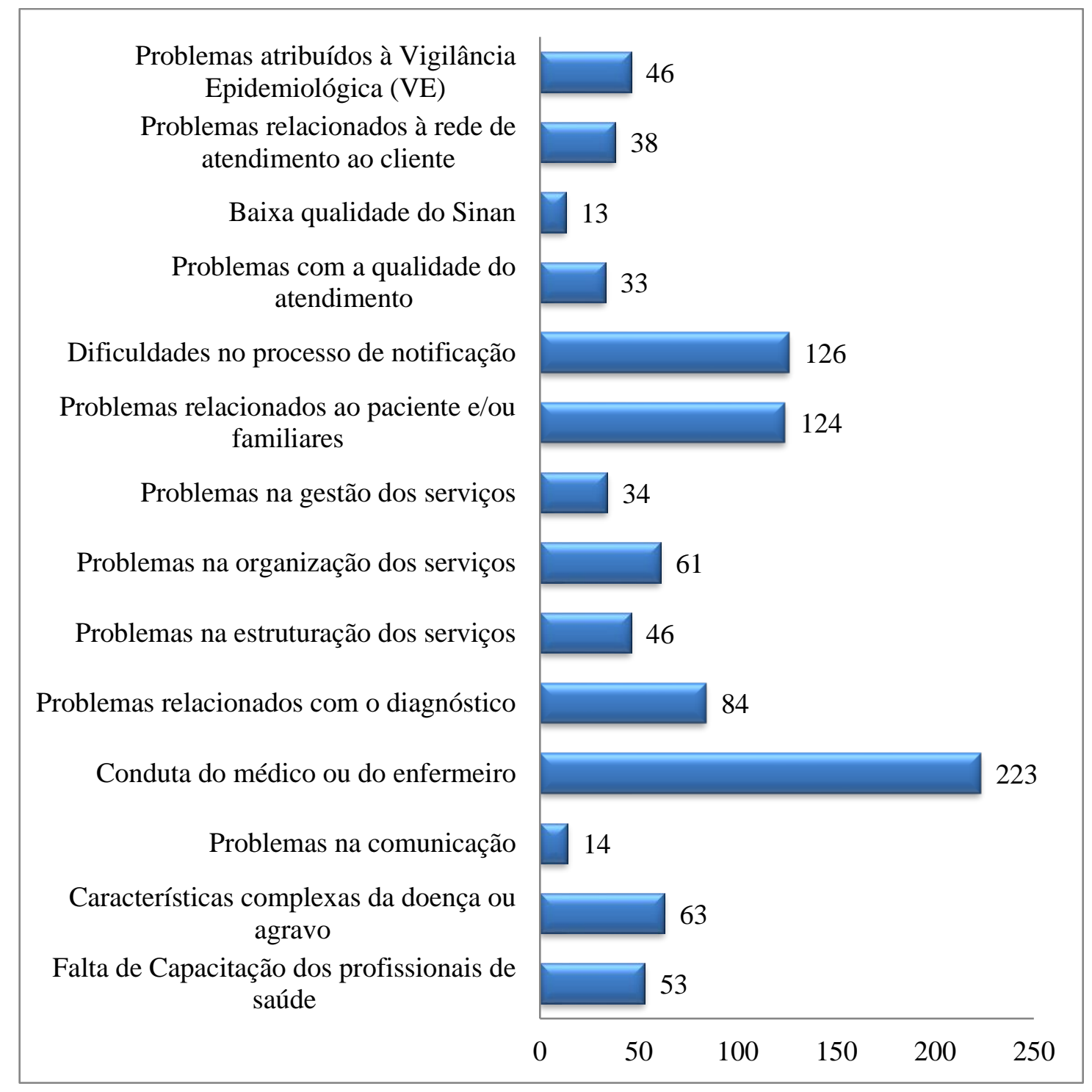

Figura 1. Resultados da categorização das entrevistas na dimensão "Percepções dos entrevistados sobre os fatores associados às situações dificultadoras", segundo as categorias de análise $(n=14)$ e respectivo quantitativo de menções nas situações relatadas $(n=958)$.

A análise dos itens que compõem as categorias relacionadas aos dados com maiores números de relatos nas entrevistas, na dimensão "Percepções dos entrevistados sobre os fatores associados às situações dificultadoras", possibilita retirar algumas informações que podem ser elencadas como os principais fatores que contribuem para a subnotificação no Sinan. Na categoria "Conduta do médico ou do enfermeiro", encontrou-se que os médicos não notificam $(20,6 \%)$, a notificação somente foi realizada pela enfermagem $(14,3 \%)$, os médicos não repassam o caso para notificação (12,6\%) e a notificação é realizada tardiamente ou longe do paciente (12,1\%) (Figura 2). Nos fatores relatados na categoria "Dificuldades no processo de notificação", com 126 menções pelos entrevistados, estão: sistema de notificação manual ou burocrático (18,3\%); o paciente vai embora sem notificar (12,7\%); falhas no 
processo de notificação (11,1\%); elevado tempo gasto para notificar $(9,5 \%)$; notificação apenas de determinadas doenças e dependente da gravidade $(7,9 \%)$ e fichas de notificação muito extensas ou inadequadas $(7,9 \%)$. Sobre os aspectos relacionados na categoria "Problemas relacionados ao paciente e/ou familiares", com 124 menções, estão as características complexas do paciente em primeiro lugar (19,4\%), seguida da não adesão do paciente ao tratamento ou exame (13,7\%), a não adesão do paciente à notificação $(12,1 \%)$, o medo do paciente ou familiares de relatar o caso $(10,5 \%)$ e a demora do paciente em buscar atendimento $(8,9 \%)$. Na categoria "Problemas relacionados ao diagnóstico", com 84 relatos nas entrevistas, os erros nos diagnósticos (42,9\%), diagnóstico tardio (22,6\%), não realização dos exames $(15,5 \%)$ e demora na realização ou nos resultados dos exames (14,3\%) foram os que apareceram com maior frequência.

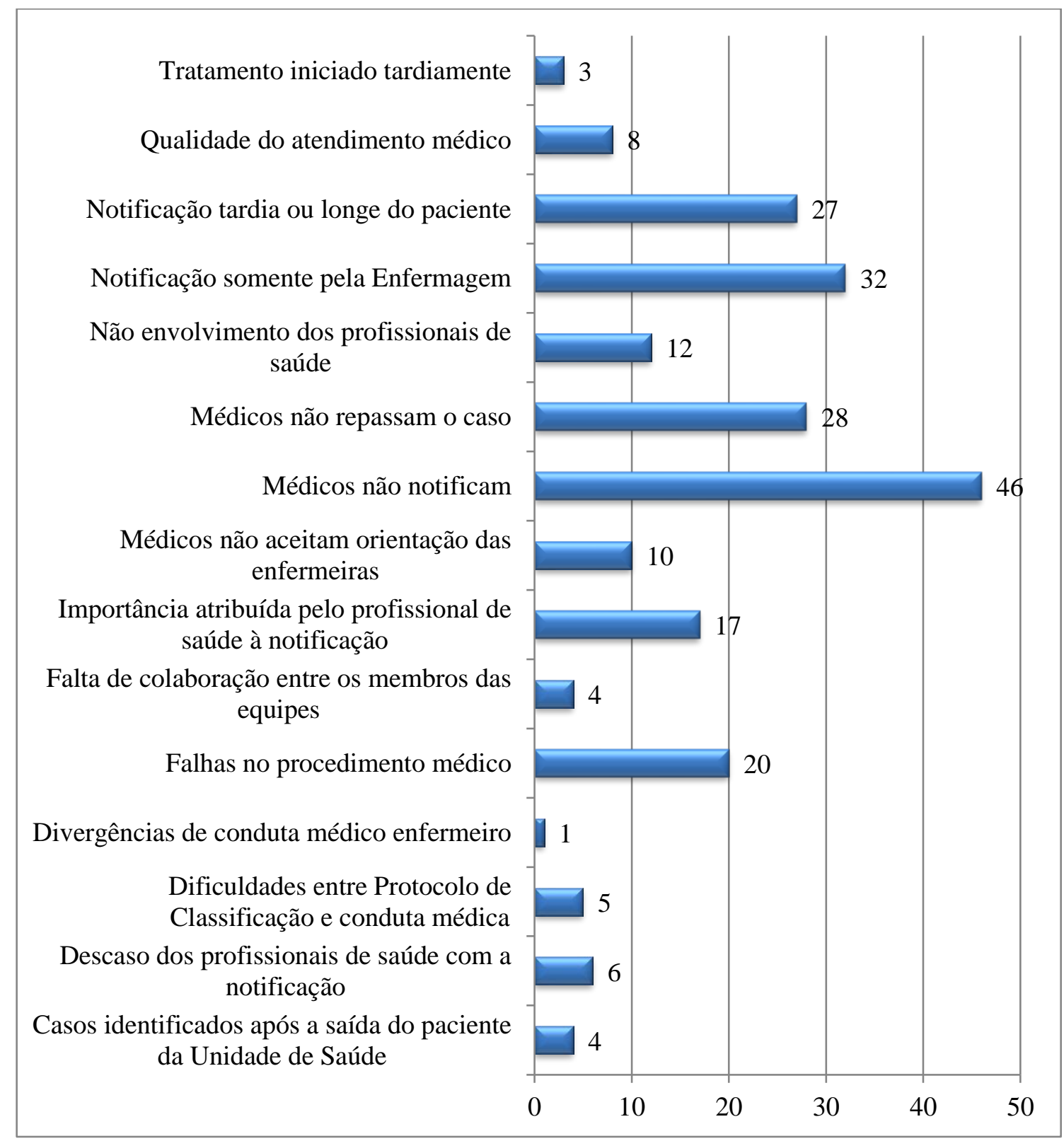


Figura 2. Resultados da categorização das entrevistas na categoria "Conduta do médico ou enfermeiro", segundo o quantitativo de menções nas situações dificultadoras relatadas e respectivos "nós" $(n=223)$.

As informações colhidas nas entrevistas são inúmeras e possibilitam diversas análises, dentre elas o estudo de correlações entre as variáveis. Realizou-se um ensaio com foco em verificar se os fatores relacionados à subnotificação no Sinan, colhidos a partir das situações relatadas, associados à categoria "Conduta do médico ou enfermeiro" ocorrem de forma generalizada nas regiões de saúde de Goiás onde o estudo se desenvolveu. Calculou-se a proporção (Pr) de citações nas situações em relação ao quantitativo de casos relatados nas entrevistas e considerou-se que valores maiores que 0,6 menções por caso seriam representados como situação ruim e que mereceria atenção dos gestores do setor. As questões dificultadoras nos processos de notificação de doenças e agravos de notificação compulsória que se relacionaram à conduta do médico ou do enfermeiro apareceram com maior intensidade nas regiões de saúde Norte $(\operatorname{Pr}=1,4)$; Pirineus $(\operatorname{Pr}=1,1)$; Entorno Sul, Estrada de Ferro, Nordeste II, Rio Vermelho e Sudoeste I (todas com $\operatorname{Pr}=0,8$ ) e, nas demais regiões, a proporção de menções ficou abaixo de 0,6. Diferentemente do esperado para a Região Central, que envolveu a capital Goiânia, com maior número de entrevistas $(n=16)$ realizadas em distintas unidades de saúde, não se verificou situação ruim para este indicador $(\operatorname{Pr}=0,5)$.

\section{DISCUSSÃO}

Houve maior quantidade de relatos de casos dificultadores, provavelmente em função das consequências que eles trazem para a população e para os serviços de saúde, principalmente quando não notificados, que neste estudo alcançou $56,4 \%$ dos comportamentos associados aos casos dificultadores relatados. Sobre a obtenção de maior quantidade de casos negativos ou positivos em estudos desta natureza, a literatura aborda que esta pode estar atrelada aos objetivos do estudo e que há tendência de os brasileiros evitarem o envolvimento com casos negativos, não os descrevendo para evitar consequências futuras dos seus relatos, mesmo com as garantias do anonimato dos respondentes formalizadas nos protocolos de pesquisa ${ }^{16}$.

Os casos mais referidos pelos entrevistados provavelmente refletem a situação epidemiológica da população amostral, já que os mais relatados envolviam a Dengue, Acidente por animal potencialmente transmissor da Raiva, Hanseníase, a Sífilis, Síndrome Respiratória Aguda - SRAG, Hepatites, Tuberculose e Meningites. Como exemplo desta situação, citam-se: a Dengue que é problema crescente de saúde no Brasil22, com as maiores taxas de incidência de casos prováveis na Região Centro-Oeste $(88,3$ casos por 100 mil habitantes e, em Goiás, essa taxa sobe para 151,7 casos por 100 mil habitantes) ${ }^{23}$; Sífilis em gestante que tem aumento de prevalência e, consequentemente, a incidência de sífilis congênita que é crescente, em função de fragilidades dos serviços de saúde que possuem falhas no 
diagnóstico, acompanhamento e tratamento dos casos ${ }^{24}$; a taxa de mortalidade por tuberculose nas capitais brasileiras que ainda é importante problema de saúde pública, com 2,7 óbitos por 100 mil habitantes e uma das principais causas de morte no conjunto das doenças transmissíveis em adulto ${ }^{25}$. Sobre os acidentes por animais potencialmente transmissores da raiva, no Brasil, houve procura anual de atendimento por exposição ao vírus da raiva, em média, 425.400 pessoas, no período $2000-2009$. Destas, $64 \%$ receberam o tratamento pós-exposição, no entanto os casos de raiva humana notificados no período 1999-2009 somaram 218 , de maneira que $66 \%$ deles não tiveram tratamento profilático e os motivos foram o desconhecimento do paciente sobre a necessidade da profilaxia ou a falta de acesso aos serviços de saúde ${ }^{26}$.

Os fatores que contribuem para a subnotificação estão atrelados, principalmente, à conduta do médico ou enfermeiro, às dificuldades no processo de notificação, às características complexas do paciente e/ou familiares e ao diagnóstico da doença. Mas o principal problema das subnotificações parece ser que os médicos têm dificuldades para diagnosticar os casos, não notificam, não repassam para outros profissionais notificar, e quando notificado é conduzida sob a responsabilidade exclusiva da equipe de enfermagem e longe do paciente, portanto, tardiamente. Essa dificuldade no diagnóstico poderá estar associada à qualidade da formação profissional ou à estrutura inadequada das unidades de atendimento ao paciente que poderão refletir na qualidade do trabalho, o que carece de melhor análise. Por outro lado, a notificação que é legalmente de responsabilidade de todos os profissionais de saúde, quando não realizada, não gera quaisquer penalidades àqueles profissionais que deixam de comunicar às autoridades de saúde os casos ocorridos. A diversidade de atribuições e a sobrecarga de trabalho dos profissionais de saúde parecem ser a principal "desculpa" para a não notificação, conforme apontado em $21,8 \%$ dos casos relatados.

Os resultados encontrados neste estudo evidenciam problemas que persistem no cotidiano das unidades de atendimento ao paciente, como, por exemplo, a notificação considera atividade burocrática ${ }^{27}$ ou sem importância pelos profissionais de saúde ${ }^{28}$; a sobrecarga dos profissionais de saúde ${ }^{27}$; a falta de confirmação diagnóstica das doenças ${ }^{9}$; as características complexas do paciente e as falhas na vigilância epidemiológica da doença ${ }^{29}$; e os problemas nos registros médicos no atendimento ${ }^{30}$.

\section{CONSIDERAÇÕES FINAIS}

A subnotificação ou a notificação tardia não contribui para as ações de vigilância quando da necessidade de bloqueio endêmicos, assim como aquelas realizadas sem a presença do paciente que tendem a ser incompletas e não terem seguimento, o que torna o trabalho de notificação dificultoso e inócuo, com reflexos na vigilância epidemiológica. Observou-se que há grande esforço da Vigilância em Saúde em realizar a notificação das doenças e agravos de notificação compulsória, seja através da equipe de enfermagem ou dos Núcleos de Vigilância, pois dos casos facilitadores relatados $(n=111)$ apenas $4,5 \%$ foram notificados pelo médico. 
Em face do volume de dados gerados neste estudo e reconhecendo a importância dos achados para a vigilância em saúde, tendo em vista que não são passíveis de apresentação em apenas um artigo, os resultados gerais do estudo foram apresentados aos gestores da Vigilância em Saúde do Estado de Goiás. Como continuidade dos trabalhos, propõe-se a construção e validação de um instrumento para medida dos níveis de adequação aos processos de notificação de doenças e agravos de notificação compulsória no Sinan para uso no Sistema Único de Saúde.

Considera-se que os objetivos propostos foram alcançados, na medida em que se identificaram os principais fatores que contribuem para a subnotificação no Sinan, obtidos por meio da condução de estudo em uma a amostra representativa da população em face dos critérios estabelecidos na respectiva estratificação.

Em termos de limitações deste estudo, relatam-se as possíveis subjetividades na formulação das categorias e na interpretação dos casos ou situações que são superadas pela possibilidade de se trabalhar com fatos que puderam ser explorados pelos pesquisadores para garantir níveis elevados de "objetividade, representatividade, realismo, aplicabilidade e utilidade" 16 .

Espera-se que estes achados possam servir de base para o estabelecimento de políticas e planos para a área da vigilância em saúde, na busca de encontrar alternativas que melhorem os processos de trabalho e ofereçam condições para que aqueles que são legalmente obrigados a notificar doenças e agravos de notificação compulsória no Sinan, o façam de maneira oportuna e adequada. Reconhecer que a notificação é importante para o sistema de vigilância em saúde e que os dados gerados com maior fidedignidade se constituem instrumento de melhoria da qualidade da saúde da população constitui-se em grande desafio do setor. É preciso que os gestores se dediquem a superar as deficiências existentes nas unidades de atendimento, onde as doenças e os agravos surgem, ao mesmo tempo em que a proteção da saúde da população dependerá da forma como os profissionais de saúde irão receber e tratar essas informações.

\section{REFERÊNCIAS}

1. Brasil. Ministério da Saúde. Secretaria de Vigilância em Saúde. Departamento de Vigilância Epidemiológica. Guia de vigilância epidemiológica. 7. ed. Brasília: Ministério da Saúde; 2009.

2. Brasil. Constituição da República Federativa do Brasil: 1988. Brasília: Câmara dos Deputados; 2003.

3. Brasil. Presidência da República. Lei no 8.080, de 19 de setembro de 1990. Dispõe sobre as condições para a promoção, a proteção e a recuperação da saúde, a organização e o funcionamento dos serviços correspondentes, e dá outras providências. Diário Oficial da República Federativa do Brasil, Brasília (DF), 1990 set 20; Seção 1:18055. 
4. Oliveira CM, Cruz MM. Sistema de Vigilância em Saúde no Brasil: avanços e desafios. Saúde em Debate 2015; 39(104): 255-67.

5. Brasil. Ministério da Saúde. Portaria no 204, de 17 de fevereiro de 2016. Define a Lista Nacional de Notificação Compulsória de doenças, agravos e eventos de saúde pública nos serviços de saúde públicos e privados em todo o território nacional, nos termos do anexo, e dá outras providências. Diário Oficial da República Federativa do Brasil, Brasília (DF), 2016 fev 18 n. 32; Seção 123-24.

6. Brasil. Ministério da Saúde. Secretaria de Vigilância em Saúde. Departamento de Vigilância Epidemiológica. Sistema de Informação de Agravos de Notificação - Sinan: normas e rotinas. 2. ed. Brasília: Editora do Ministério da Saúde; 2007.

7. Oliveira MTDC, Barreira D, Santos LCO, Latorr MRDO. A subnotificação de casos de aids e municípios brasileiros selecionados: uma aplicação do método de captura-recaptura. Bol Epidemiol AIDST 2004; 18(1): 7-11.

8. Pillaye J, Clarke A. An evaluation of completeness o tuberculosis notification in the United Kingdom. BM Public Health 2003; 3(31). DOI:10.1186/1471-2458 3-31.

9. Santos ML. Fatores associados à subnotificação de TB e Aids, durante os anos de 2001 a 2010, a partir do Sinan [dissertação de mestrado]. Recife (PE): Centro de Pesquisas Aggeu Magalhães, Fundação Oswaldo Cruz; 2014.

10. Ciríaco DL. Subrregistro de óbitos por aids: investigação de óbitos por causas indeterminadas ou com diagnósticos sugestivos de imunodeficiência adquirida na região metropolitana de Maceió, Alagoas [dissertação de mestrado]. Recife (PE): Centro de Pesquisas Aggeu Magalhães, Fundação Oswaldo Cruz; 2010.

11. Komka MR, Lago EG. Sífilis congênita: notificação e realidade. Sci Med 2007; 17(4): 205-11.

12. Serapioni M. Métodos qualitativos e quantitativos na pesquisa social em saúde: algumas estratégias de integração. Cienc Saude Colet 2000; 5(1): 187-92.

13. Selltiz C, Wrightsman LS, Cook SW. Métodos de pesquisa das relações sociais. São Paulo: Herder; 1965.

14. Mattar FN. Pesquisa de marketing. 3 ed. São Paulo: Atlas; 2001.

15. Lakatos EM, Marconi MA. Fundamentos metodologia científica. 4. ed. São Paulo: Atlas; 2001.

16. Dela Coleta JA, Dela Coleta MF. A técnica dos incidentes críticos: 30 anos de utilização no Brasil na Psicologia, Administração, Saúde e Educação. Taubaté-SP: Cabral Editora e Livraria Universitária; 2004. 
17. Franco MLPB. Análise do conteúdo. Brasília: Liber Livro; 2008.

18. Bardin L. Análise de conteúdo. Tradução de Luís Antero Reto; Augusto Pinheiro. São Paulo: Edições 70; 2016.

19. Roesch SMA. Projetos de estágio e de pesquisa em administração: guia para estágios, trabalhos de conclusão, dissertações e estudos de caso. Colaboração Grace Vieira Becker e Maria Ivone de Mello. 3. ed. São Paulo: Atlas; 2005.

20. Instituto Brasileiro de Geografia e Estatística. Número de municípios e população nos Censos Demográficos, segundo as classes de tamanho da população dos municípios - Goiás - 1960/2010 [Internet]. [citado 20 jun 2017]. Disponível em:

http://www.imb.go.gov.br/viewcad.asp?id_cad=450.

21. Brasil. Conselho Nacional de Saúde. Resolução № 466, de 12 de dezembro de 2012. Aprova as diretrizes e normas regulamentadoras de pesquisas envolvendo seres humanos. Diário Oficial de República Federativa do Brasil, Brasília (DF), 2013 jun 13; Seção 1:59.

22. Martelli CMT, Siqueira Junior JB, Parente MPPD, Zara AL, Oliveira CS, Braga C, et al. Economic Impact of Dengue: Multicenter Study across Four Brazilian Regions. PLoS Negl Trop Dis, 2015; 9(9): e0004042. DOI:10.1371/journal.pntd.0004042.

23. Brasil. Ministério da Saúde. Secretaria de Vigilância em Saúde. Monitoramento dos casos de dengue, febre de chikungunya e febre pelo vírus Zika até a Semana Epidemiológica 10, 2017. Boletim Epidemiológico 2017; 48(9): 1-10.

24. Cavalcante PAM, Pereira RBL, Castro JGD. Sífilis gestacional e congênita em Palmas, Tocantins, 2007-2014. Epidemiol Serv Saude 2017;26(2): 255-64.

25. Ceccon RF, Maffacciolli R, Burille A, Meneghel SN, Oliveira DLLC, Gerhardt TE. Mortalidade por tuberculose nas capitais brasileiras, 20082010. Epidemiol Serv Saude 2017; 26(2): 349-58.

26. Brasil. Ministério da Saúde. Secretaria de Vigilância em Saúde. Departamento de Vigilância Epidemiológica. Normas técnicas de profilaxia da raiva humana. Brasília: Ministério da Saúde; 2011.

27. Barreto PA, Braga ALS, Andrade M. Avaliação da completitude dos registros de dengue: estudo exploratório das notificações compulsórias. Online Braz J Nurs 2012; 11(3): 21.

28. Emmerick ICM, Campos MR, Schramm JMA, Silva RS, Costa MFS.

Estimativas corrigidas de casos de meningite, Brasil 2008-2009. Epidemiol Serv Saúde 2014; 23(2): 215-26. 
29. Sousa LMO, Pinheiro RS. Óbitos e internações por tuberculose não notificados no município do Rio de Janeiro. Rev Saúde Pública 2011; 45(1): 31-9.

30. Escosteguy CC, Medronho RA, Madruga R, Dias HG, Braga RC, Azevedo OP. Vigilância epidemiológica e avaliação da assistência às meningites. Rev Saúde Pública 2004; 38(5):657-63.

Recebido: 15 de abril de 2018. Publicado: 05 de junho de 2018

Correspondência: Maria Aparecida de Souza Melo. E-mail: cida.souza.melo@gmail.com

Conflito de Interesses: os autores declararam não haver conflito de interesses.

(C) This is an Open Access article distributed under the terms of the Creative Commons Attribution License, which permits unrestricted use, distribution, and reproduction in any medium, provided the original work is properly cited 